\title{
A New Approach for RFID Tag Data Reading in FPGA by using UART and FIFO
}

\author{
Pavan Ambati ${ }^{\mathrm{a}}$, Mrudula Singamsetti ${ }^{\mathrm{b}}$ \\ ${ }^{a}$ Vignan'S University, Andhra Pradesh, INDIA \\ ${ }^{b}$ Vignan'S University, Andhra Pradesh, INDIA
}

Received: 21 June 2017; Accepted: 11 September 2017; Published: 08 March 2018

\begin{abstract}
Nowadays, Radio Frequency Identification (RFID) technology used for automatic object tracking and identifying purpose. RFID technology used in so many applications especially in automobiles, security and health care systems. RFID reader and RFID tags are main processing units in RFID system. RFID tags give the information on what an object is, where it is and even its condition, and what is happening, share related data and respond to the reader. RFID reader module used for reading data from lots of RFID tags. In convention methods [1][2] doesn't contain any read controller methodology and doesn't contain any storage element for RFID tags data storing. In this paper, we propose a new approach for RFID tag data reading in FPGA. This approach is more flexible in structure and easily updates the design for any applications. This design is easily adaptable for different chips and communication mechanism. This design has reading controlling methodology and along with storing capability of RFID tags by using FIFO. Here we are using Universal Asynchronous Receiver and Transmitter protocol for communicating RFID reader to PC. For simulation, we are using ModelSim software and for synthesis, purpose using XILINX ISE 14.7.
\end{abstract}

Index Terms: RFID reader, RFID Tag, UART, FIFO, FPGA, VERILOG, ModelSim, XILINX ISE 14.7.

(C) 2018 Published by MECS Publisher. Selection and/or peer review under responsibility of the Research Association of Modern Education and Computer Science.

\section{Introduction}

Radio Frequency Identification Technology (RFID) is a wireless technology used for automatic object identifying. This technology uses the radio magnetic waves for transferring data between RFID tag and RFID reader. RFID technology is the replacement of a Barcode system and there is no physical contact between the

* Corresponding author. Tel.: +91-9160832981; Tel:+91-8328697232

E-mail address: abpavan3@gmail.com, mrudulasingamsetti@gmail.com 
Tag and Reader. RFID system mainly contains two processing units [3][4]. They are

\section{- RFID TAG}

- Active Tag

- Passive Tag

\begin{tabular}{|ll|}
\hline Nomenclature \\
RFID & Radio Frequency Identification \\
UART & Universal Asynchronous Receiver and Transmitter \\
FIFO & First In First Out \\
UID & Unique Identification Number \\
FPGA & Field Programmable Gate Array \\
\hline
\end{tabular}

\section{- RFID READER}

RFID Tags are attached to the object and that tags tell what an object is, where it is and its condition. RFID tag contains storing element. Storage element contains the object details. RFID tags are classified as the Active tag and Passive Tag. Active Tag contains a battery for transmitting data to the reader. Active Tag is communicated the longer distances but it is costly. A passive tag doesn't contain any battery. RFID Passive Tag active when power is received from RFID reader. Passive tag communicates smaller distance and it is cheaper. A reader sent power source to tag through electromagnetic induction coupling principle. Induction coupling means to transfer the energy from one circuit to another circuit through electromagnetic waves. A RFID tag contains Unique Identification Number (UID) for identifying the object details. This UID number is read by the RFID reader and sends the UID in serial form by using UART. RFID system transfer data between the reader and Tag without any physical contact. Data was transmitted through radio frequency waves. It will operate the radio frequency at low-frequency between $125 \mathrm{kHz}-134 \mathrm{KHz}$ and high frequency at $13.56 \mathrm{MHz}$ and Ultra High-frequency range from $860 \mathrm{MHz}-960 \mathrm{MHz}$ [4] Fig 1 gives the information on RFID communication system.

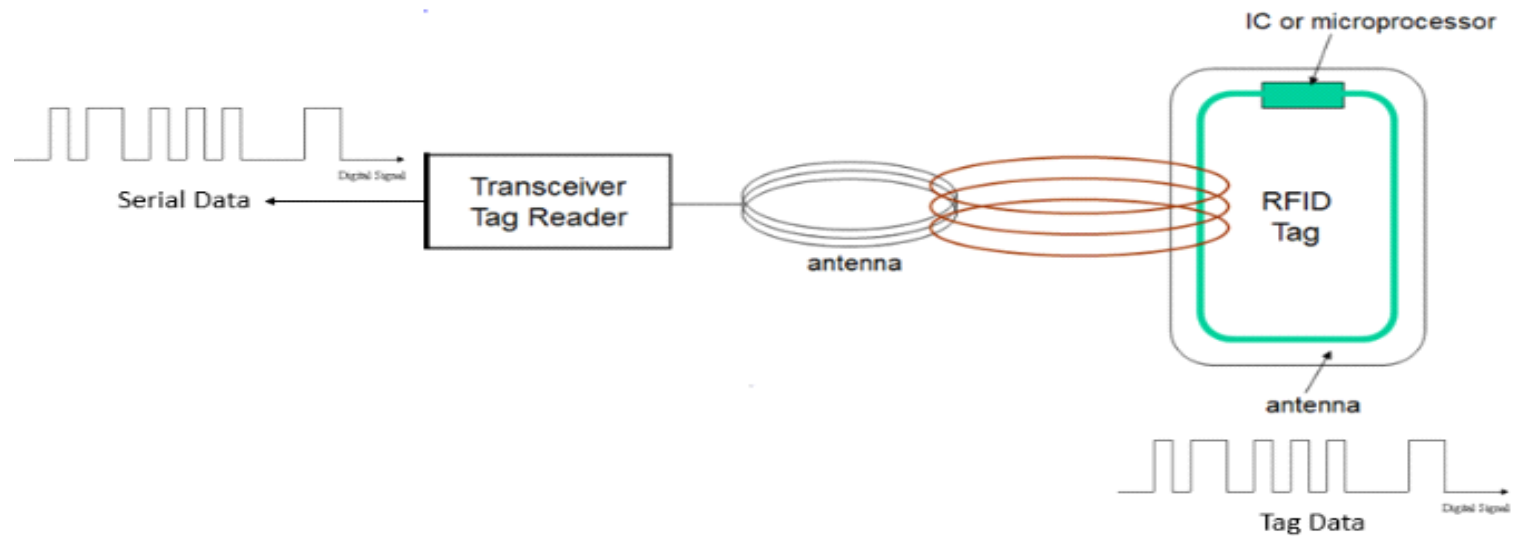

Fig.1. RFID Communication System 


\section{Previous Approach and Proposed Method}

\subsection{Existing Method}

In convention method approaches [1][2], RFID tag data reading was done by using serial communication and with the help of PC. In this process, there is no read controller module. This process is only for checking RFID tags are working or not and doesn't contain any storage of RFID tags further uses. Fig 2 shows the block diagram of RFID tag reading. In this figure RFID tag data is read by using MFRC500 (RFID reader module) and sends the data to the pc through UART interfacing. This approach is not applicable for storing RFID tag data.

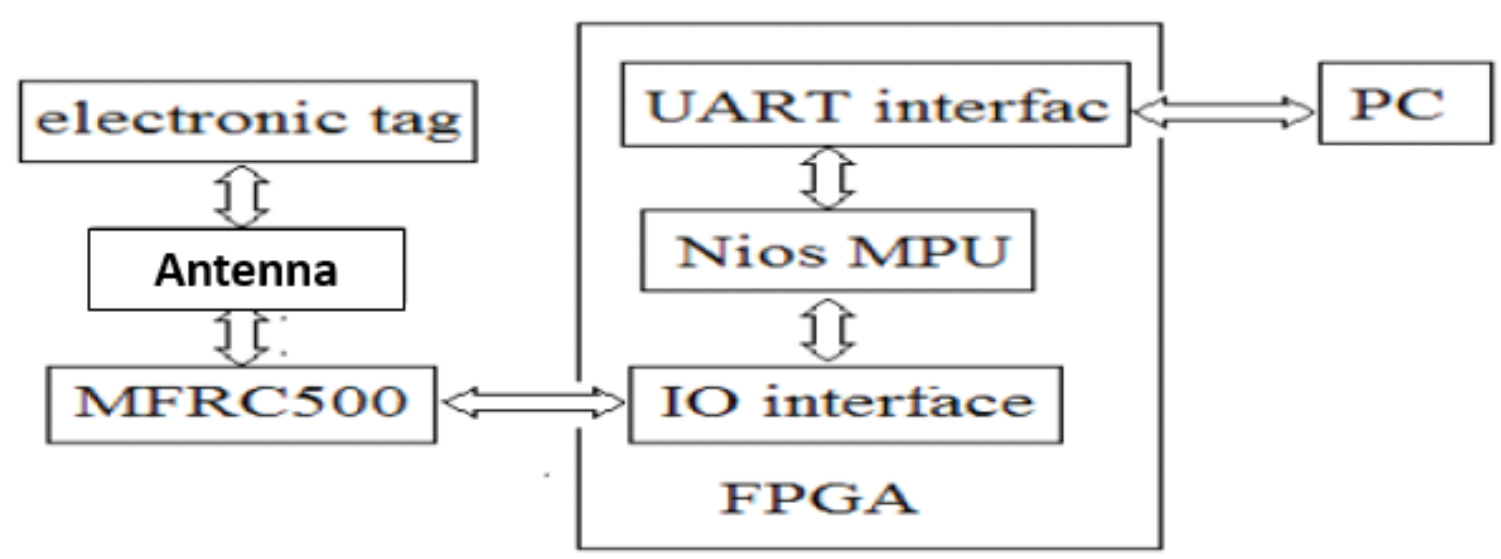

Fig.2. Previous Approach for RFID Tag Data Reading

\subsection{Proposed Method}

Fig 3 shows the proposed method for RFID tag data reading in FPGA. The proposed method is flexible in the structure and easily adaptable for different chips and communication protocols. This module contains the reader controller module for further development. This module stores RFID tags data by using FIFO (First in First Out). This module can update easily. These module works as follows

- When RFID tag comes near the RFID reader module it will reads the information from the RFID tag.

- Sends the Data in the serial format by using UART communication protocol.

- UART receiver reads the data in serial form sends Byte format to the Data Management module.

- Data Management module checks the received data send data to the write module. This module writes the data into the FIFO.

- FIFO means First In First Out.

- After writing data into the FIFO data will be read by using read controller module.

- UART transmitter module sends the data in the serial form.

- After read controller module we can place any other decoding circuit for application development and any other display monitoring system. 


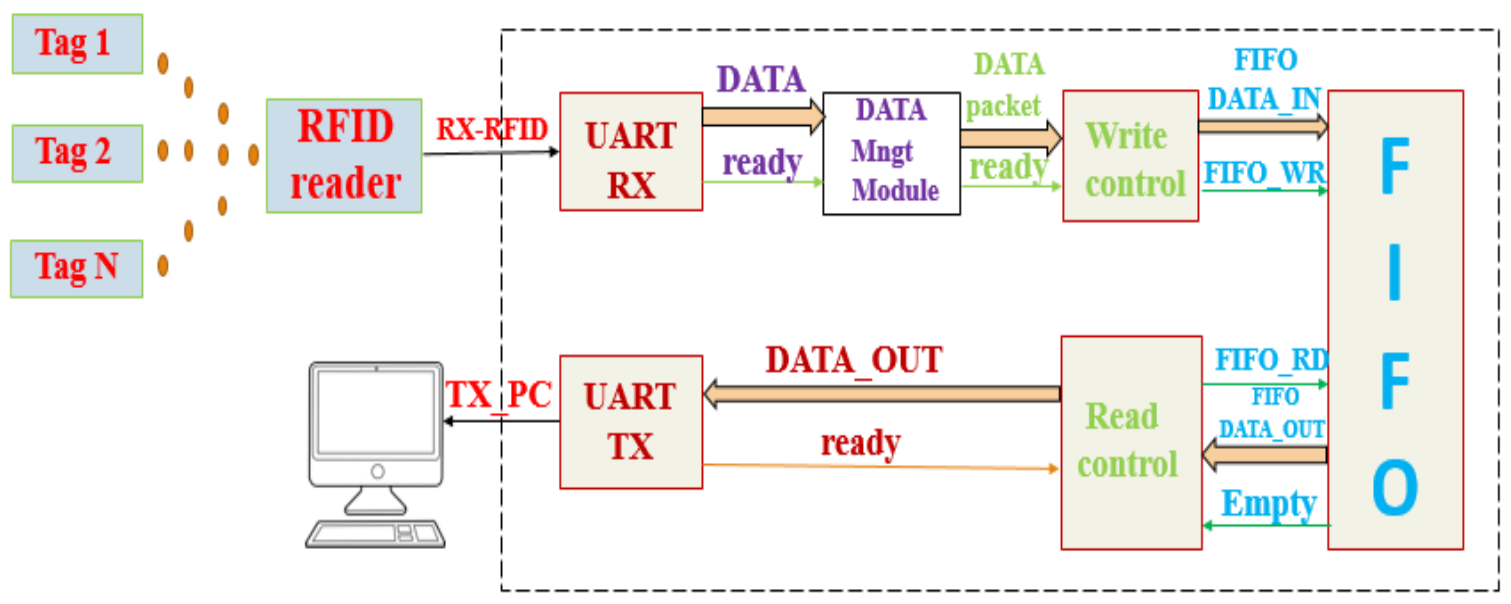

Fig.3. Proposed Block Diagram for RFID Tag Data Reading in FPGA

\section{Internal Block Explanation}

\subsection{Universal Asynchronous Receiver and Transmitter}

UART is an Asynchronous communication system that means data will not depend on the clock signal. UART is serial communication and used a single wire for transmitting/receiving data. UART has some standard configuration parameters; they are Baud rate $(2400,4800,9600 \ldots)$, start bit, data bits, stop bit, a parity bit and flow control. Before going to the communication establishment, we need to fix these parameters as same as in receiver and transmitter. Fig 4 indicates the UART data format. UART contains receiver and transmitter modules. In these design, we are taken configuration parameters as baud rate (9600), data bits (8bits), a start bit (1-Bit), stop bit (1bit) and no flow control, no parity bit.

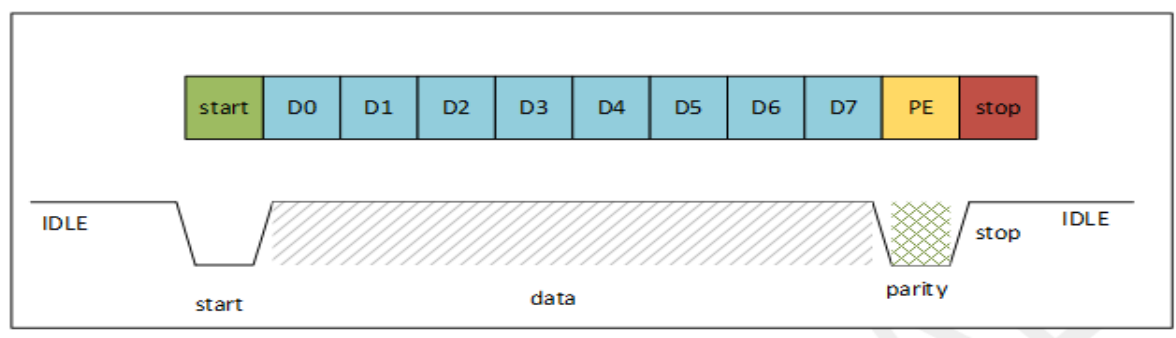

Fig.4. Data Framing

\subsubsection{UART Receiver}

UART receiver module receives data in the serial form and output in parallel form. In this module, we use simple shift registers for getting parallel data. UART receiver module is designed by using FSM. Data was taken at the middle of samples in the data frame. This module is active when serial data is the active low position. Fig 5 and fig 6 shows the block diagram of UART receiver module and output waveform respectively. 


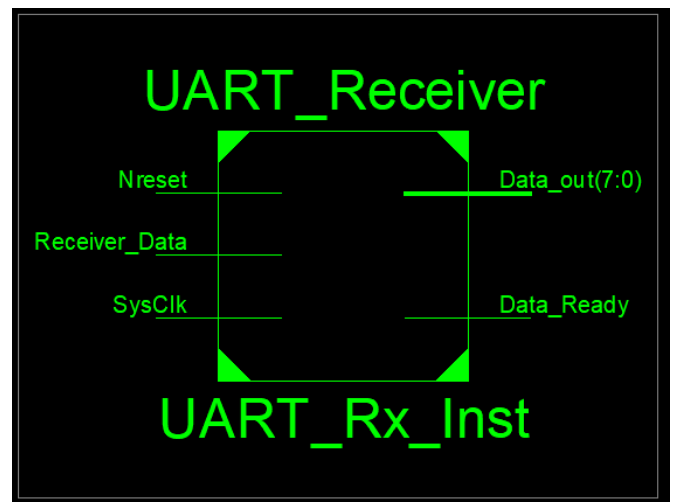

Fig.5. Block Diagram of UART Receiver module

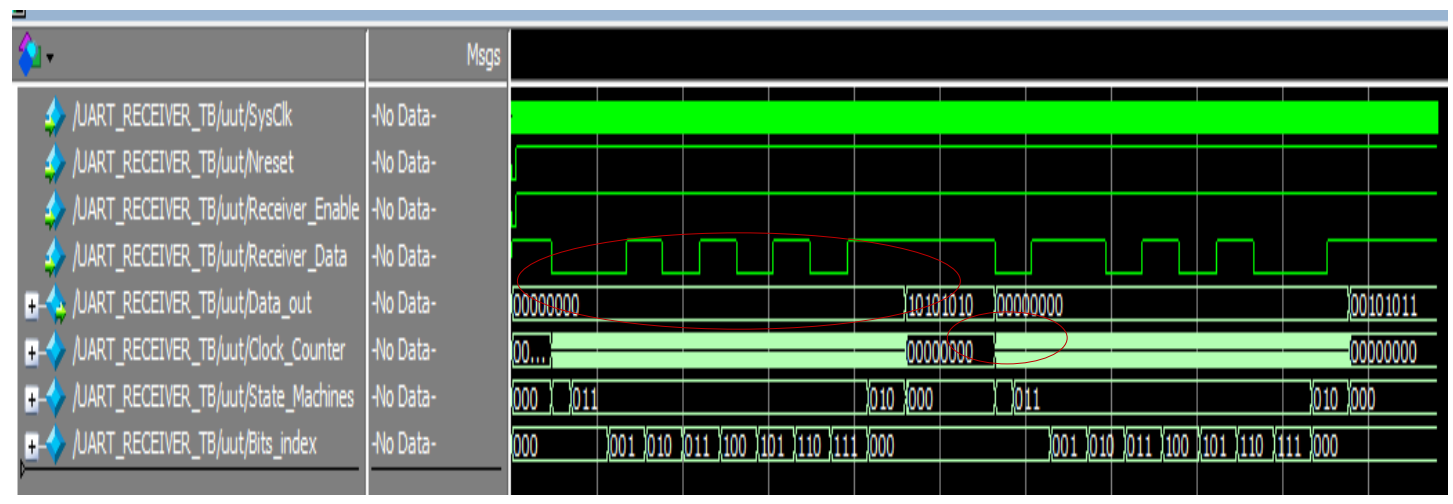

Fig.6. Output waveform of UART Receiver

\subsubsection{UART Transmitter}

UART transmitter module works same as the shift register. UART transmitter module design follows same as the UART receiver module. In this module, data will be shifted serially. Fig 7 and Fig 8 shows the block diagram of UART transmitter and output waveform. These module receives the data in the form of parallel and sends data into the serial.

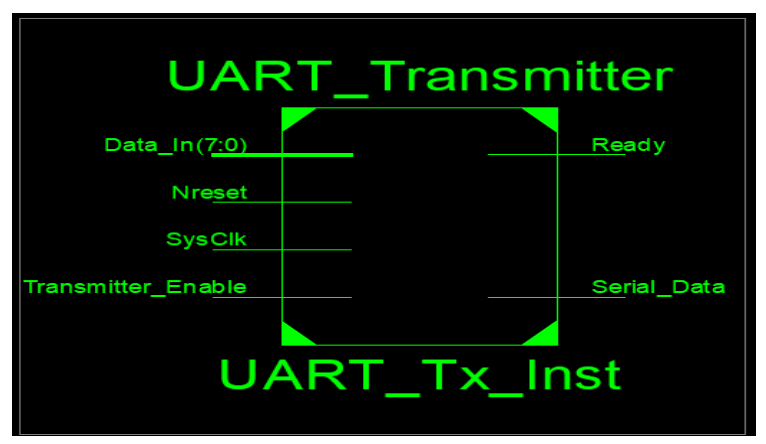

Fig.7. Block Diagram of UART Transmitter Module 


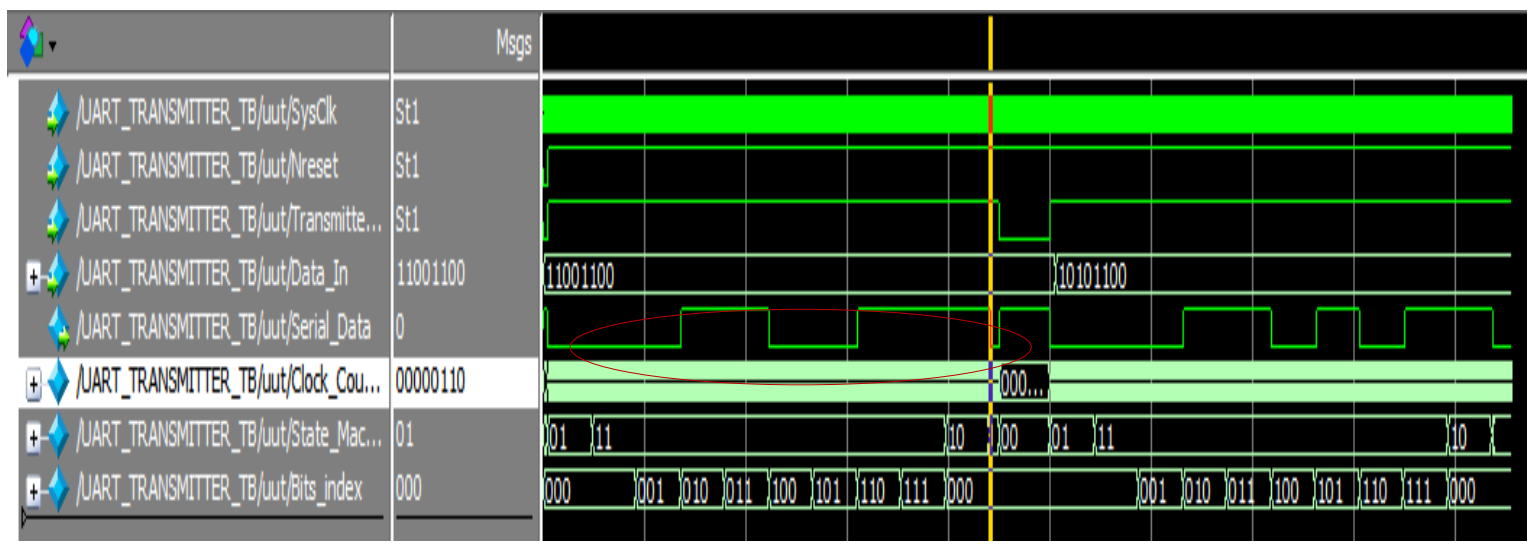

Fig.8. Output waveform of UART Transmitter

\subsection{Internal Module}

\subsubsection{Data Management System}

UART receiver module outputs are connected to the Data Management System module. This module receives the parallel data from the UART receiver module. This module taken Byte data from UART receiver module and checks received data correct or not. This module checks the start byte (0x0A) of RFID tag and stops byte (0x0D) of RFID tag. If the start and stop byte is correct then RFID tag data packet sent to the write controller module. Data Management System module reduces the mismatch of RFID tag data and gives the accurate result as the output. For this module using simple shift registers, comparators. Outputs of Data Management System module is connected to the write controller module. Fig 9 and Fig 10 gives the block diagram of data management module and output waveform respectively.

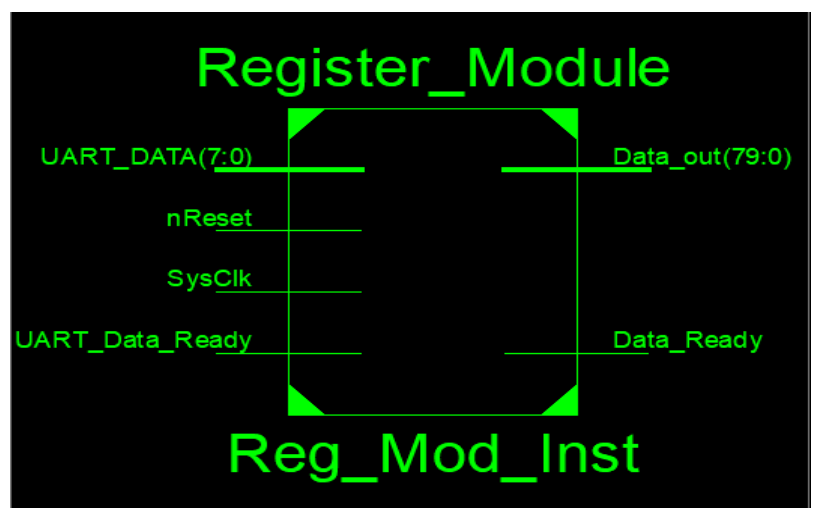

Fig.9. Block Diagram of Data Management Module 


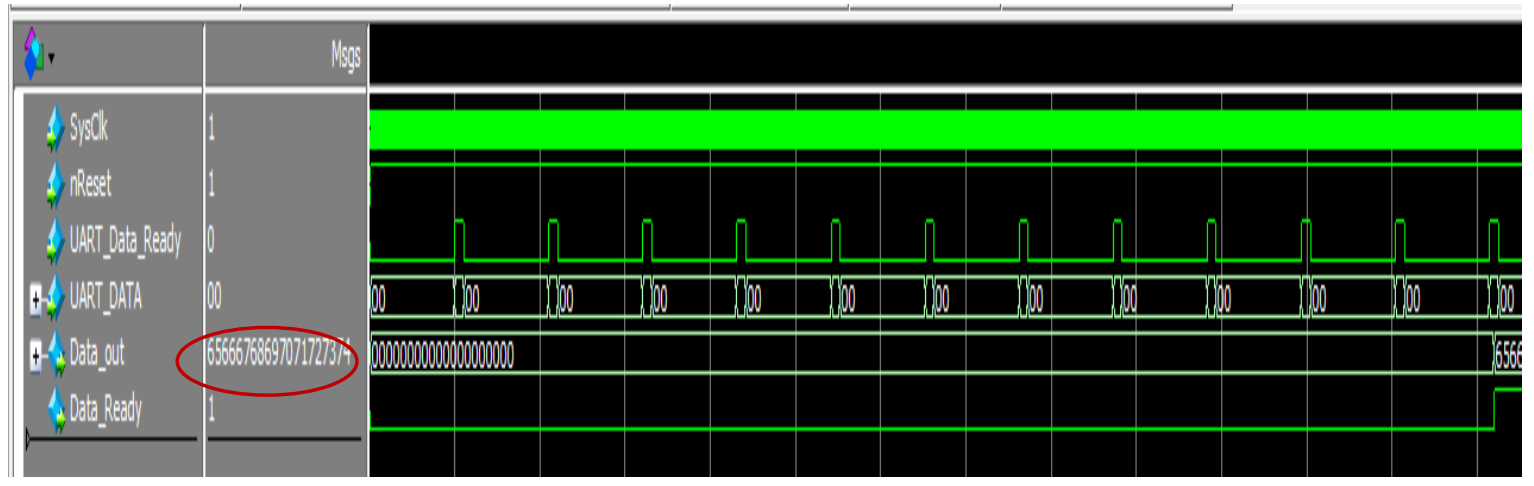

Fig.10. Output waveform of Data Management Module

\subsubsection{Write Controller}

Write controller module is used for writing data into the FIFO. When Ready goes high it will read RFID data packet and send 8bit data every 3 clock signals and sends write signal on second clock signal due to this data will stable when writing happens into the FIFO. Fig 11 indicates the block diagram of write controller module. Fig 12 shows the data written into the FIFO.

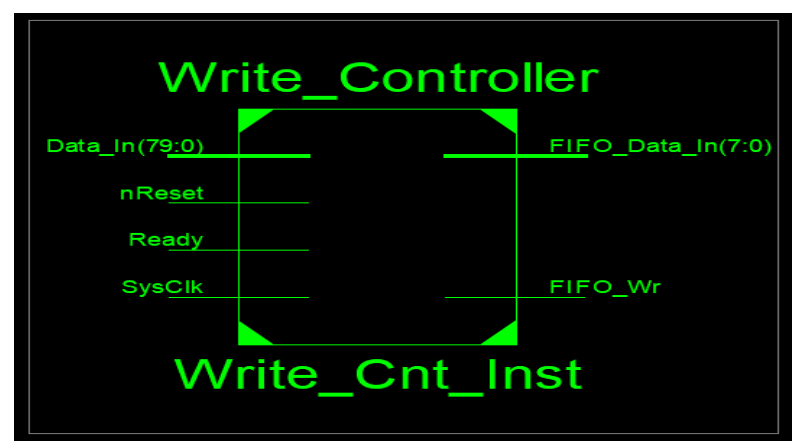

Fig.11. Block Diagram of Write Controller Module

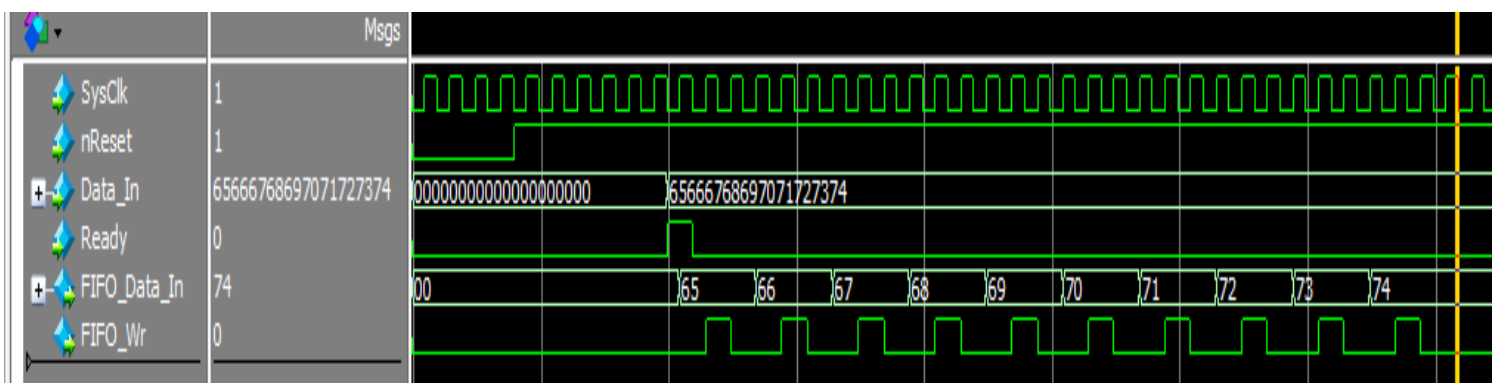

Fig.12. Data is Written into the FIFO 


\subsubsection{READ CONTROLLER}

Read controller sends the read signal to the FIFO and reads the data from the FIFO. Read controller module sends Byte data to the UART transmitter module. This module also receives a ready signal from UART transmitter module for indicating data is transmitted in the serial form. This module also makes an important role in RFID tag reading. This module active when FIFO contains any data. By using this module we can develop any application. For developing application require UID number. These numbers stored in the FIFO and these data read by using read controller module. Fig 13 and 14 indicates the block diagram and the output waveform of reading controller module respectively.

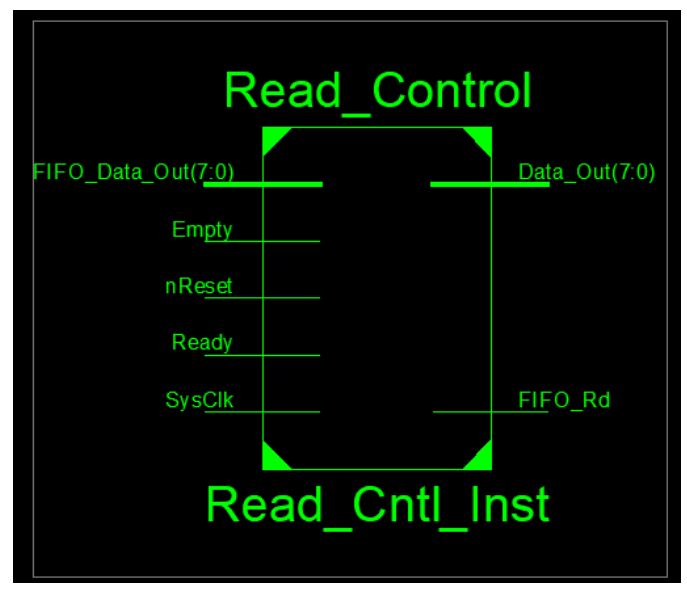

Fig.13. Block Diagram of Reading Controller Module

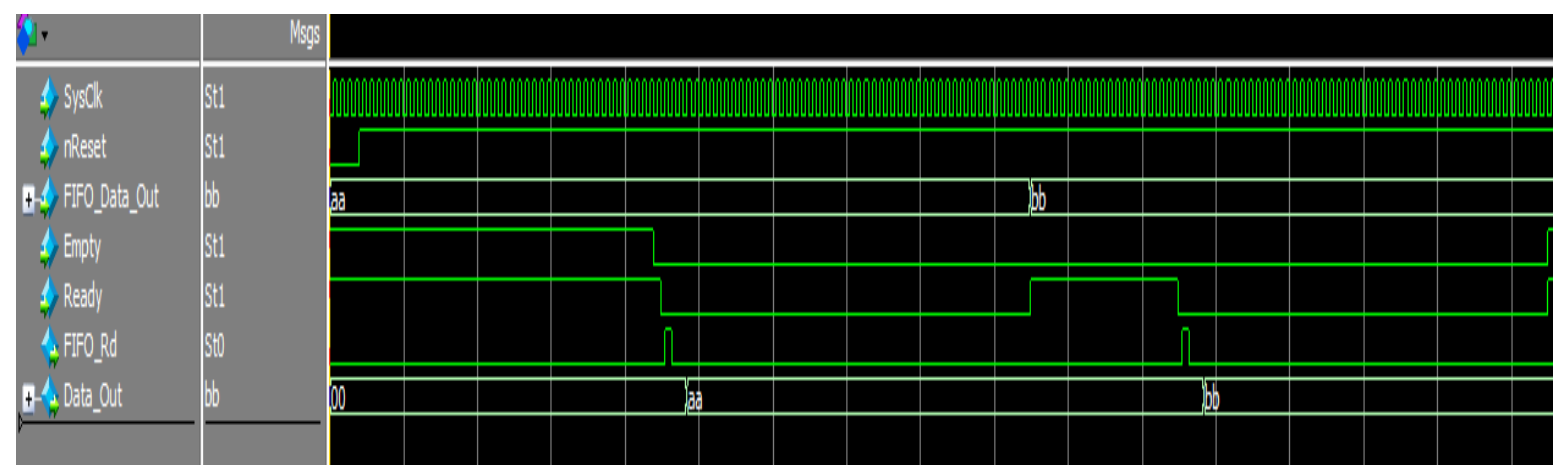

Fig.14. Output waveform of Reading Controller Module

\section{Experimental Results}

RFID reader reads the information from RFID tag through radio frequency waves and reader will send the data in serial form. RFID reader sends start byte (0x0A), data bytes (10 Bytes) and stop byte (0x0D) respectively. Fig 15 indicates the RTL schematic view of the proposed method. Fig 16 shows the data receiving from RFID reader in serial form. 


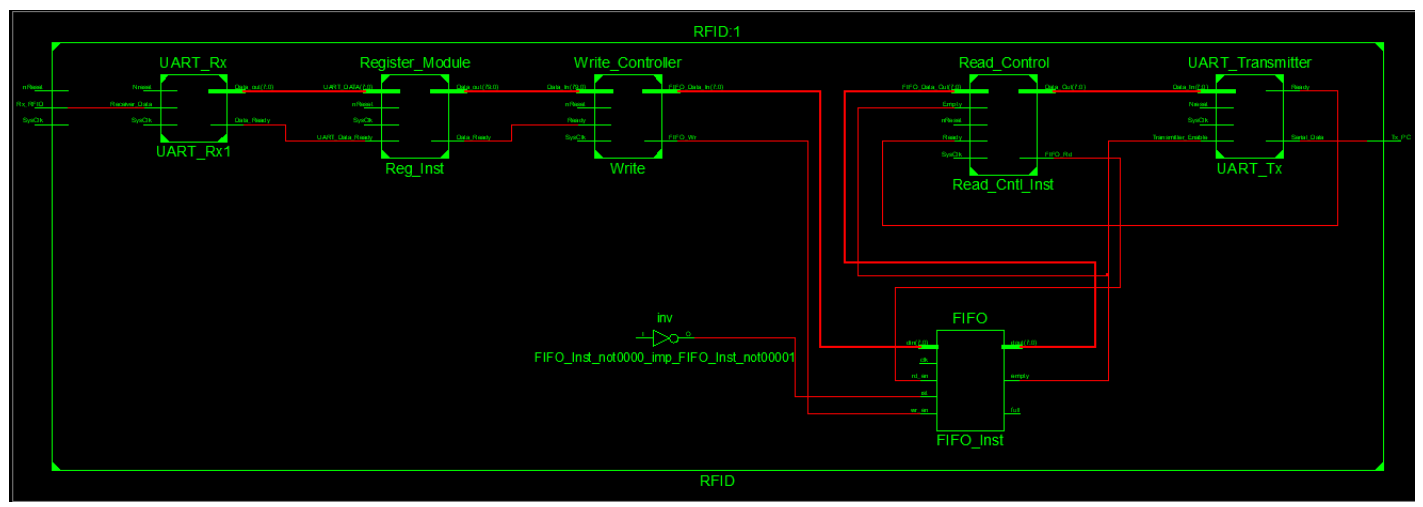

Fig.15. Rtl Schematic of Proposed Method

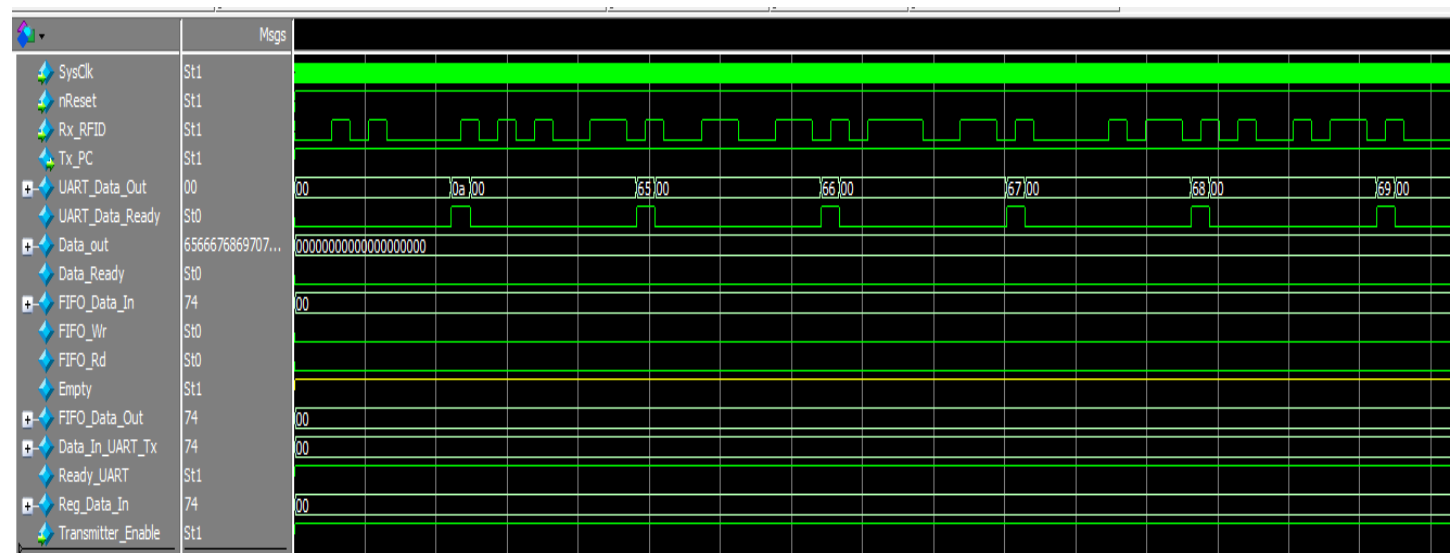

Fig.16. Reading the Information from RFID Tag

UART Receiver receives data in serial and sends in byte format. Data management system checks receiving correct data or not and sends a data packet to the write controller module. This module writes the byte data into FIFO. Fig 17 shows the write data into the FIFO.

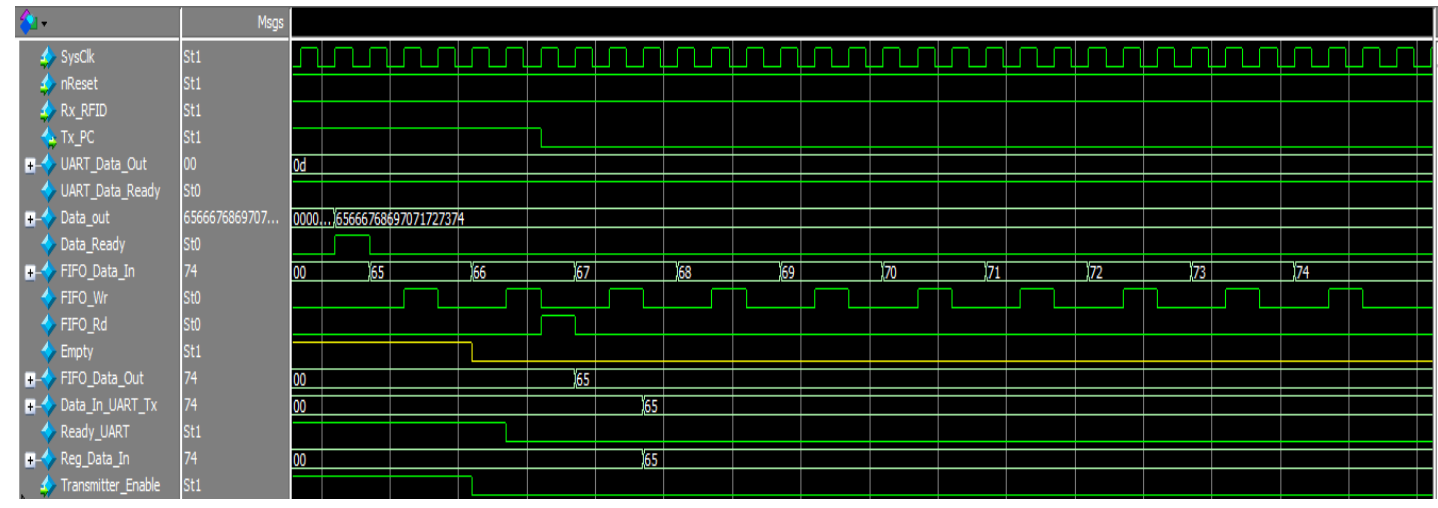

Fig.17. Writing Data into the FIFO by Write Controller 
Read controller module reads the data from FIFO and sends to UART transmitter module. This module receives data in the parallel form and sends in serial form. Fig 18 shows the sends data in serial form by UART Transmitter.

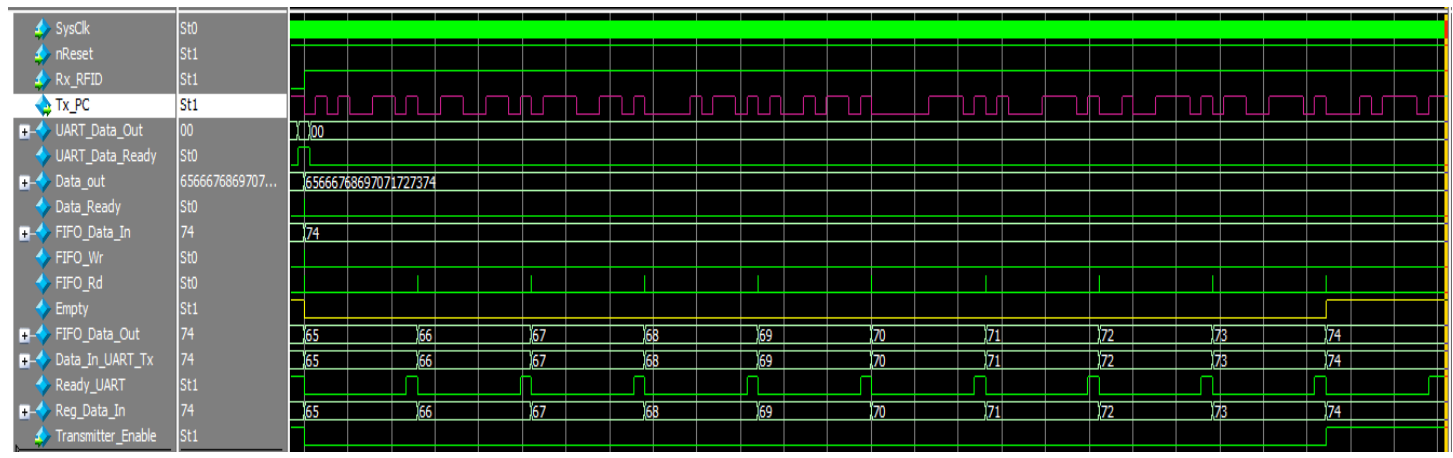

Fig.18. Sending Data to the PC through serial Communication

Table 1. Difference between Existing Method and the Proposed Method

\begin{tabular}{ccc}
\hline Parameters & Existing Method & Proposed method \\
\hline Adaptable for Different communications & YES & YES \\
Adaptable for different Chips & YES & YES \\
Update available & YES & YES \\
Storage capability & NO & YES \\
Read Controller & NO & YES \\
Application development & NOT POSSIBLE & POSSIBLE \\
IP-core generation & NO & YES \\
\hline
\end{tabular}

With the help of Hyper Terminal, we can see the RFID tag data. Table 1 gives information difference between existing method and the proposed method. The proposed method is applicable for application development and fig 19 shows the development of IP core. Table 2 shows the experimental results of the proposed method and executed in the Xilinx ISE 14.7. Maximum PATH DELAY: 2.184ns

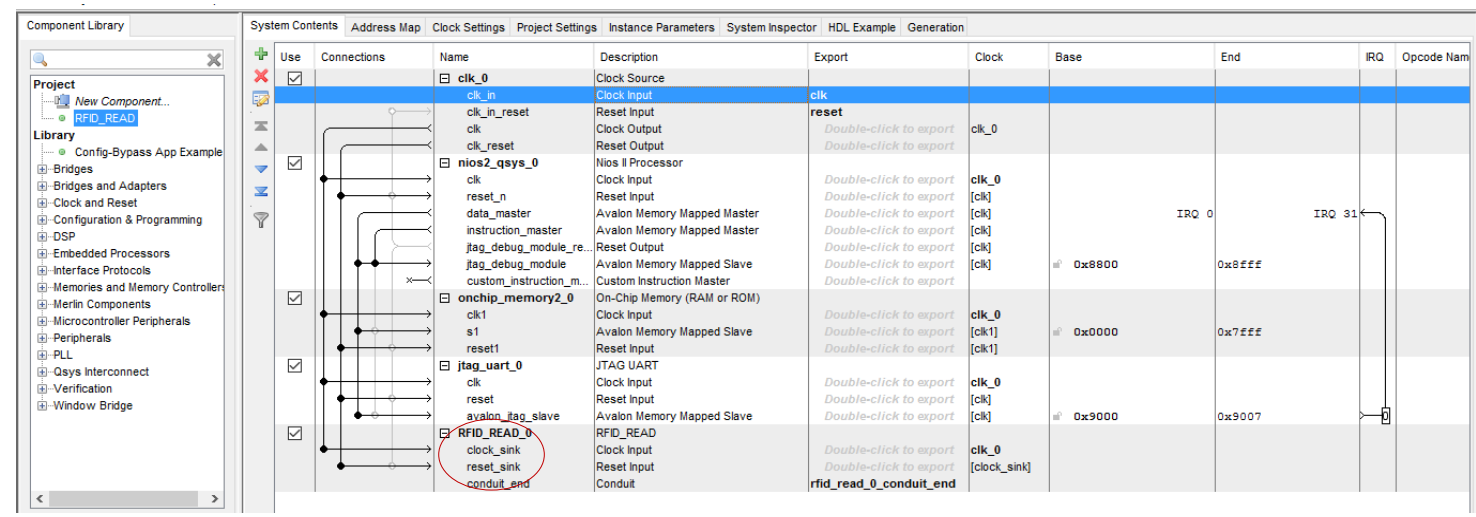

Fig.19. IP core Generation for RFID Tag Reading 
Table 2. Simulations Results of Proposed Method

\begin{tabular}{|l|r|r|r|r|}
\hline \multicolumn{4}{|c|}{ Device Utilization Summary (estimated values) } & Utilization \\
\hline Logic Utilization & Used & Available & 4656 & $6 \%$ \\
\hline Number of Slices & 316 & 9312 & $4 \%$ \\
\hline Number of Slice Flip Flops & 418 & 9312 & $4 \%$ \\
\hline Number of 4 input LUTs & 375 & 232 & $1 \%$ \\
\hline Number of bonded IOBs & 4 & 1 & 20 & $5 \%$ \\
\hline Number of BRAMs & 1 & 24 & $4 \%$ \\
\hline Number of GCLKs & & & \\
\hline
\end{tabular}

\section{Conclusion}

In previous approaches, RFID tag data reading was done by using serial communication and with the help of PC. In previous approaches, there is no read controller module. Previous process is only for checking RFID tags are working or not and doesn't contain any storage of RFID tags further uses. Proposed method is flexible in the structure and easily adaptable for different chips and communication protocols. This module contains the reader controller module for further development. This module stores RFID tags data by using FIFO (First in First Out). This module can update easily. This module also useful for application development process.

\section{References}

[1] Wenping Ren, Chulin Shao, Aimin Miao, Jiqin He "A FPGA -based processing Circuit and Realization for Baseband signals of RFID”2016IEEE international conference held in MAY. DOI:10.1109/ICOACS2016. 7563108.

[2] Neelappa, Dr.N.G. Kurahatti "Novel Method for design and implementation of low power FPGA based RFID System" 2015 IEEE international conference held in March.DOI:10.1109/ICECCT.2015.7226143

[3] G.Rajeshwari and V.D.M. Jabez Daniel "FPGA realization of RFID Tag Data Reading Enhancement Mechanism by Using Parallel Processing" Middle-East Journal of Scientific Research 24 (4):1330-1334, 2016 April.

[4] Rahul Varma, Zoonubiya Ali “A Brief Overview of RFID Tag Design in FPGA”, IJSR, Volume 5 Issue 5 , May 2016.

[5] Zsolt Kemeny, Elisabeth IceTudor, Laszlo Monostori and Peter Egri, 2014. "The RFID Technology and its current applications" IEEE Transactions on Electron Devices, 61(7).

[6] He Fu gui,Hou Yi bin. Li Hui, "Realizati- of RFID reader based on FPGA" Journal of Lioaoning Technical University ,Feb 2009.

[7] Laszlo Monostori, Elisabeth lie zudor, Zsolt Kemeny and Peter Egri, 2014 "The RFID Technology and its current applications" IEEE Transactions on Electron Devices.61(7).

[8] Yuanqing Zheng, Mo Li, Yuanqing, Limo, Geng Shu-qin, Wu-Chen, Hou Li-gang and Zhang Wang,2015. "Read Bulk Data from Computational RFID", IEEE Transactions on Industrial Electronics, 62(7).

[9] NSK electronics RFID reader $125 \mathrm{Khz}$ datasheet web-http://www.nskelectronics.in-files/rfid_edk_kit.pdf.

[10] Sridhar Iyer, "RFID:Technology and Applications”, IIT Bombay ,Presentation, 2005.

[11] Behzard Razavi, "RF Microelectronics”, Dorling Kindsley India pvt.ltd., 2013.

[12] Marlin H. Mickle, Swapna R. Dontharaju, Shenchih Tung,Ralph Sprang, Raymond R. Hoare, Josh Fazekas, James T. Cain, and Alex K. Jones, “An Automated, Reconfigurable, Low-Power RFID Tag”, DAC 2006, July 24-28, 2006,pp 131-136. 
[13] Jianping Wang Huiyun Li and Fengqi Yu, "Design of Secure and Low-cost RFID Tag Baseband", 14244- 1312-5/07 @ 2007 IEEE, pp. 2066-2069.

[14] Su-Bong Ryu, Jin-Oh Jeon, and Min-Sup Kang, "FPGA Design of Digital Codec for Passive RFID Tag", 2007 IEEE DOI 10.1109/ALPIT.2007.99, pp. 343-346.

[15] Yujing Feng, Wei Zhang, Xiaohui Xing, "Digital part in 915MHz UHF RFID tag", 978-1-4244-40764/09/ (2009 IEEE, pp. 115-119.

\section{Authors' Profiles}

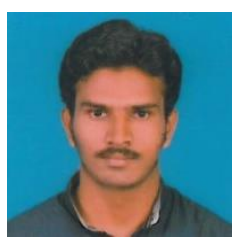

AMBATI PAVAN received his Bachelor's Degree in Electronics and Communication Engineering from Vignan's University, Andhra Pradesh, India in the year 2015 and obtained his Master's Degree in VLSI Design from Vignan's University, Andhra Pradesh, India in the year 2017. He is working as the Verification Engineer from June 2016. His Research interest includes also in Digital Circuits, FPGA and Verification Engineering.

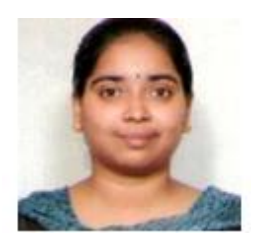

Ms.Mrudula Singamsetti was born in Moturu(A.P.) India. She received the B.Eng.degree in electronics and communication engineering from Raja Lakshmi Engineering College, Chennai, India, in 2007, the M.Tech. degree in VLSI System Design in VNR Vignana Jyothi Institute of Engineering \& Technology,Hyderabad India, in 2009.She is currently working as an Assistant Professor with the Department of Electronics and Communication Engineering, Vignan's Foundation for Science Technology and Research University. Her research area includes Quantum Cellular Automata, Digital Circuits, VLSI testing and verification.

How to cite this paper: Pavan Ambati, Mrudula Singamsetti,"A New Approach for RFID Tag Data Reading in FPGA by using UART and FIFO", International Journal of Engineering and Manufacturing(IJEM), Vol.8, No.2, pp.33-44, 2018.DOI: 10.5815/ijem.2018.02.04 